\title{
Near-Optimal Dynamic Trajectory Generation and Control of an Omnidirectional Vehicle.
}

\author{
Tamás Kalmár-Nagy, Raffaello D’Andrea, Pritam Ganguly \\ Sibley School of Mechanical and Aerospace Engineering \\ Cornell University \\ Ithaca, NY 14853, USA
}

April 8, 2002

\begin{abstract}
This paper describes a computationally inexpensive, yet high performance trajectory generation algorithm for omnidirectional vehicles. It is shown that the associated nonlinear control problem can be made tractable by restricting the set of admissible control functions. The resulting problem is linear with coupled control efforts and a near-optimal control strategy is shown to be piecewise constant (bang-bang type). A very favorable trade-off between optimality and computational efficiency is achieved. The proposed algorithm is based on a small number of evaluations of simple closed-form expressions and is thus extremely efficient. The low computational cost makes this method ideal for path planning in dynamic environments.
\end{abstract}

\section{Introduction}

Omnidirectional vehicles provide superior maneuvering capability compared to the more common nonholonomic (car-like) vehicles. The ability to move along any direction, irrespective of the orientation of the vehicle, make it an attractive option in dynamic environments. The annual RoboCup competition, where teams of fully autonomous robots engage in soccer matches, is an example of where omnidirectional vehicles have been used in computationally intensive, dynamic environments (Kitano, 1997, Asada and Kitano, 1998, Veloso et al., 1999, D'Andrea et al., 2001).

Most papers on trajectory control of omnidirectional vehicles have dealt with relatively static environments (Moore and Flann, 1999, Jung et al., 1999); the trajectory control is essentially performed by first building a geometric path and then by using feedback control to track the path. This strategy is effective when reaching the goal without collisions is much more important than time-optimality. In fast paced environments, however, the dynamic capabilities of the vehicles must be taken into account. Muñoz et al. (1994) presented methods for planning mobile robot trajectories by considering kinematic and dynamic constraints on the motion of the vehicle. Faiz and Agrawal (2000) recently proposed a trajectory planning scheme for open-chain systems that takes the dynamics of the system into account, as well as inequality constraints. Watanabe et al. (1998) demonstrated that with a resolved-acceleration type feedback full omnidirectionality can be achieved with decoupled rotational and translational motion. A novel trajectory generation method based on a time-scaled artificial potential field was put forth by Tanaka et al. (1998). 
The objective of this paper is to establish a real-time control strategy that will move the robot to a given location, with zero final velocity, as quickly as possible, based on measurements of the vehicle position and orientation. The results of this paper can then be used as a basis for real-time trajectory generation in dynamic environments. The success of the Cornell RoboCup team is partly due to the effectiveness of the proposed algorithm (D'Andrea et al., 2001). A subsequent paper will address how this method can be applied to a real system and will include experimental results. The organization of the paper is as follows. Section 2 describes the kinematic and dynamic model of the omnidirectional vehicle. Section 3 shows that the translational and rotational degrees of freedom (DOF) of the vehicle can be independently controlled by imposing constraints on the control efforts. Section 4 describes the construction of one-dimensional minimum time and fixed time trajectories as well as the solution to the relaxed trajectory generation problem. Simulations are presented in Section 5. The paper ends with some concluding remarks in Section 6.

\section{Kinematic and Dynamic Modeling of the Omni- directional Vehicle}

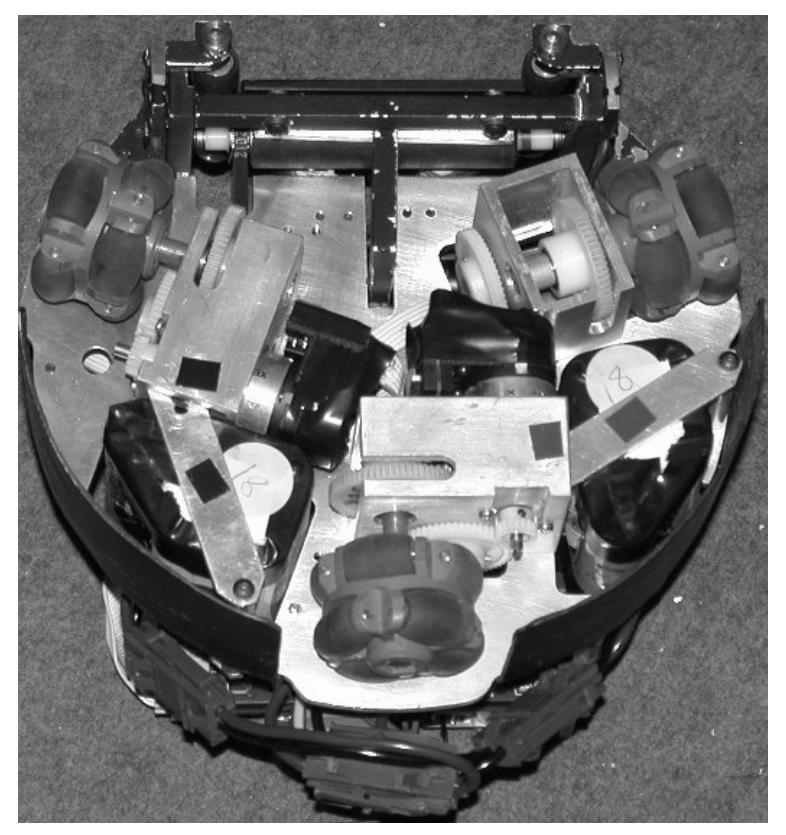

Figure 1: Bottom view of the omnidirectional vehicle

The omnidirectional drive consists of three sets of wheel assemblies equally spaced at 120 degrees from one another (see Figure 1). Each of the wheel assembly consists of a pair of "orthogonal wheels" (Pin and Killough, 1994) with an active (the propelling direction of the actuator) and a passive (free-wheeling) direction which are orthogonal to each other. The point of symmetry is assumed to be co-incident with the center of mass (CM) of the robot.

\subsection{Vehicle Kinematics}

The schematic arrangement of the wheel assemblies is shown in Figure 2. 


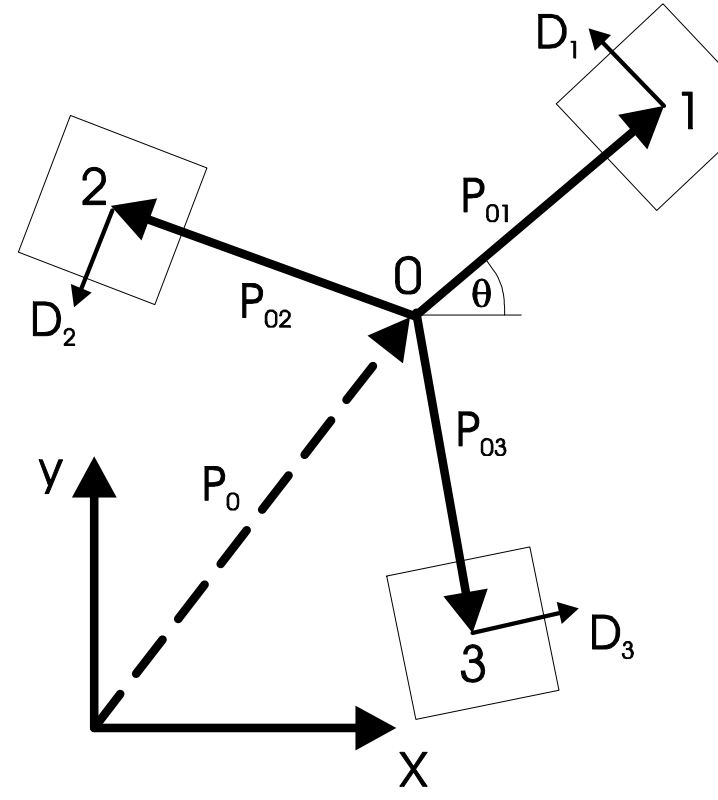

Figure 2: Geometry of the omnidirectional vehicle

The positions $\left(\mathbf{P}_{0 i}\right)$ of these units are easily given (in the frame which is fixed to the center of mass of the robot) with the help of the rotation matrix ( $\theta$ is the angle of counterclockwise rotation)

$$
\mathbf{R}(\theta)=\left(\begin{array}{cc}
\cos \theta & -\sin \theta \\
\sin \theta & \cos \theta
\end{array}\right)
$$

as

$$
\begin{gathered}
\mathbf{P}_{01}=L\left(\begin{array}{c}
1 \\
0
\end{array}\right) \quad \mathbf{P}_{02}=\mathbf{R}\left(\frac{2 \pi}{3}\right) \mathbf{P}_{01}=\frac{L}{2}\left(\begin{array}{c}
-1 \\
\sqrt{3}
\end{array}\right) \\
\mathbf{P}_{03}=\mathbf{R}\left(\frac{4 \pi}{3}\right) \mathbf{P}_{01}=-\frac{L}{2}\left(\begin{array}{c}
1 \\
\sqrt{3}
\end{array}\right)
\end{gathered}
$$

where $L$ is the distance of the drive units from the CM. The unit vectors $\mathbf{D}_{i}$ that specify the drive direction the $i$ th motor (also relative to the $\mathrm{CM}$ ) are given by

$$
\begin{aligned}
\mathbf{D}_{i} & =\frac{1}{L} \mathbf{R}\left(\frac{\pi}{2}\right) \mathbf{P}_{0 i} \\
\mathbf{D}_{1}=\left(\begin{array}{c}
0 \\
1
\end{array}\right) \quad \mathbf{D}_{2} & =-\frac{1}{2}\left(\begin{array}{c}
\sqrt{3} \\
1
\end{array}\right) \quad \mathbf{D}_{3}=\frac{1}{2}\left(\begin{array}{c}
\sqrt{3} \\
-1
\end{array}\right)
\end{aligned}
$$

\subsection{The Motor Characteristics}

In general, the optimal control problem for independent actuator driven wheels is treated with either bounded velocity (Jung et al., 1999) or bounded acceleration (Renaud and Fourquet, 1997) but not both. A reasonably accurate model that captures the torque $T$ produced by a direct current (DC) motor is

$$
T=\bar{\alpha} U-\bar{\beta} \omega
$$

where $U[\mathrm{~V}]$ is the voltage applied to the motor, and $\omega[\mathrm{rad} / \mathrm{s}]$ is the angular velocity of the motor shaft. Inductance can be neglected. The motor is characterized by the constants $\bar{\alpha}$ 
$[\mathrm{Nm} / \mathrm{V}]$ and $\bar{\beta}[\mathrm{Nm} \mathrm{rad} / \mathrm{s}]$. Figure 3 shows typical torque-speed characteristics of a DC motor. These profiles are also assumed to capture losses in the transfer of torques to the wheels. The salient feature of this model is that the amount of torque available for acceleration is a function of the speed of the motor.

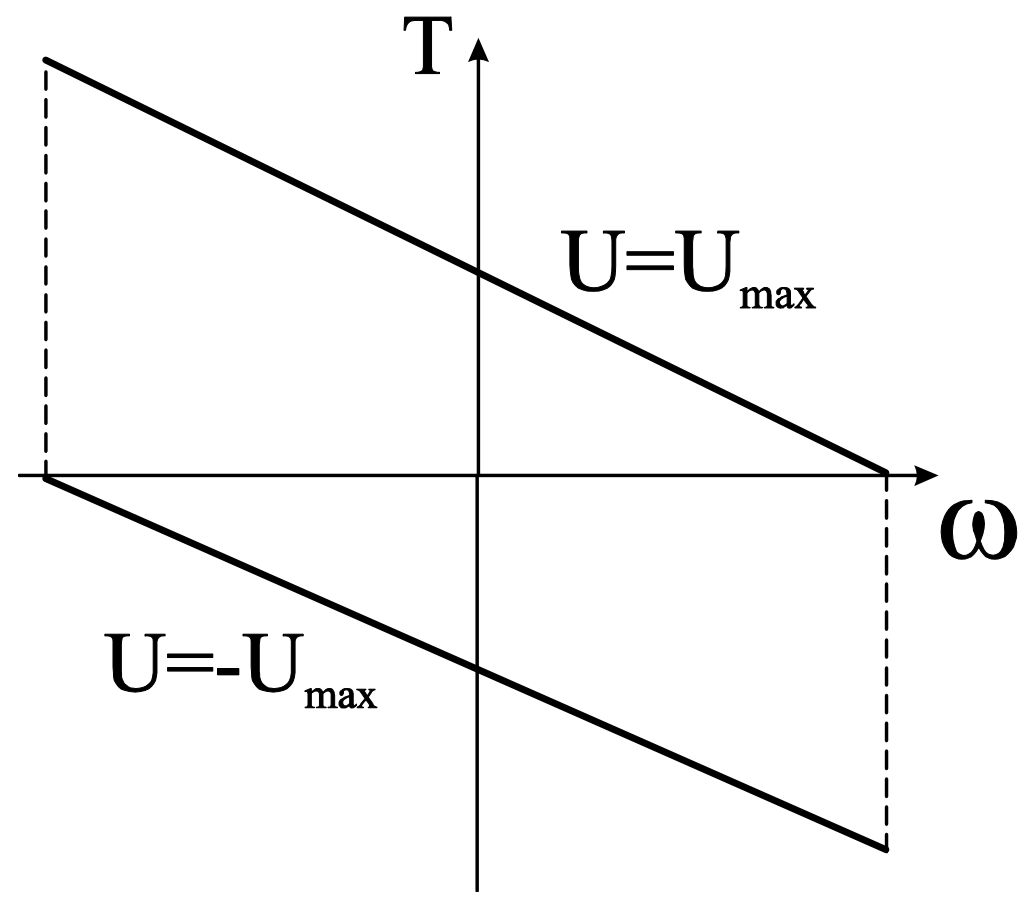

Figure 3: Torque-speed characteristics of a DC motor

With the no-slip condition, the force generated by a DC motor driven wheel is simply

$$
f=\alpha U-\beta v
$$

where $f[\mathrm{~N}]$ is the magnitude of the force generated by a wheel attached to the motor, and $v[\mathrm{~m} / \mathrm{s}]$ is the velocity of the wheel. The constants $\alpha[\mathrm{N} / \mathrm{V}]$ and $\beta[\mathrm{kg} / \mathrm{s}]$ can readily be determined from $\bar{\alpha}, \bar{\beta}$, and the geometry of the vehicle.

\subsection{Equations of Motion}

The vector $\mathbf{P}_{0}=\left(\begin{array}{ll}x & y\end{array}\right)^{\mathrm{T}}$ is the position of the CM in a Newtonian frame as shown in Figure 2. The drive positions and velocities are given by

$$
\begin{aligned}
\mathbf{r}_{i} & =\mathbf{P}_{0}+\mathbf{R}(\theta) \mathbf{P}_{0 i} \\
\mathbf{v}_{i} & =\dot{\mathbf{P}}_{0}+\dot{\mathbf{R}}(\theta) \mathbf{P}_{0 i}
\end{aligned}
$$

while the individual wheel velocities are

$$
v_{i}=\mathbf{v}_{i}^{\mathrm{T}}\left(\mathbf{R}(\theta) \mathbf{D}_{i}\right)
$$

Substituting equation (7) into equation (8) results in

$$
v_{i}=\dot{\mathbf{P}}_{0}^{\mathrm{T}} \mathbf{R}(\theta) \mathbf{D}_{i}+\mathbf{P}_{0 i}^{\mathrm{T}} \dot{\mathbf{R}}^{\mathrm{T}}(\theta) \mathbf{R}(\theta) \mathbf{D}_{i}
$$


The second term of the right hand side is just the tangential velocity

$$
\mathbf{P}_{0 i}^{\mathrm{T}} \dot{\mathbf{R}}^{\mathrm{T}}(\theta) \mathbf{R}(\theta) \mathbf{D}_{i}=L \dot{\theta}
$$

The drive velocities are thus linear functions of the velocity and the angular velocity of the robot

$$
\left(\begin{array}{l}
v_{1} \\
v_{2} \\
v_{3}
\end{array}\right)=\left(\begin{array}{ccc}
-\sin \theta & \cos \theta & L \\
-\sin \left(\frac{\pi}{3}-\theta\right) & -\cos \left(\frac{\pi}{3}-\theta\right) & L \\
\sin \left(\frac{\pi}{3}+\theta\right) & -\cos \left(\frac{\pi}{3}+\theta\right) & L
\end{array}\right)\left(\begin{array}{c}
\dot{x} \\
\dot{y} \\
\dot{\theta}
\end{array}\right)
$$

Linear and angular momentum balance can be written as

$$
\begin{aligned}
\sum_{i=1}^{3} f_{i} \mathbf{R}(\theta) \mathbf{D}_{i} & =m \ddot{\mathbf{P}}_{0} \\
L \sum_{i=1}^{3} f_{i} & =J \ddot{\theta}
\end{aligned}
$$

where $f_{i}$ is the magnitude of the force produced by the $i$ th motor, $m$ is the mass of the robot and $J$ is its moment of inertia.

Using equation (5) together with the balance laws $(12,13)$ and replacing the $v_{i}$ 's from the kinematic relation (9) results

$$
\begin{aligned}
\sum_{i=1}^{3}\left(\alpha U_{i}-\beta v_{i}\right) \mathbf{R}(\theta) \mathbf{D}_{i} & =m \ddot{\mathbf{P}}_{0} \\
L \sum_{i=1}^{3}\left(\alpha U_{i}-\beta v_{i}\right) & =J \ddot{\theta}
\end{aligned}
$$

This system of differential equations can be expressed as

$$
\left(\begin{array}{c}
m \ddot{x} \\
m \ddot{y} \\
J \ddot{\theta}
\end{array}\right)=\alpha \hat{\mathbf{P}}(\theta) \mathbf{U}(t)-\frac{3 \beta}{2}\left(\begin{array}{c}
\dot{x} \\
\dot{y} \\
2 L^{2} \dot{\theta}
\end{array}\right)
$$

with

$$
\hat{\mathbf{P}}(\theta)=\left(\begin{array}{ccc}
-\sin \theta & -\sin \left(\frac{\pi}{3}-\theta\right) & \sin \left(\frac{\pi}{3}+\theta\right) \\
\cos \theta & -\cos \left(\frac{\pi}{3}-\theta\right) & -\cos \left(\frac{\pi}{3}+\theta\right) \\
L & L & L
\end{array}\right), \quad \mathbf{U}(t)=\left(\begin{array}{c}
U_{1}(t) \\
U_{2}(t) \\
U_{3}(t)
\end{array}\right)
$$

We next introduce the new time and length scales

$$
T=\frac{2 m}{3 \beta}, \quad \Psi=\frac{4 \alpha m U_{\max }}{9 \beta^{2}}, \quad \Theta=\frac{4 \alpha m^{2} L U_{\max }}{9 J \beta^{2}}
$$

and the new nondimensional variables

$$
\bar{x}=\frac{x}{\Psi}, \quad \bar{y}=\frac{x}{\Psi}, \quad \bar{\theta}=\frac{\theta}{\Theta}, \quad \bar{t}=\frac{t}{T}, \quad \bar{U}_{i}(t)=\frac{U_{i}(t)}{U_{\max }}
$$

The nondimensional equations of motion (after dropping the bars) become

$$
\left(\begin{array}{c}
\ddot{x} \\
\ddot{y} \\
\ddot{\theta}
\end{array}\right)+\left(\begin{array}{c}
\dot{x} \\
\dot{y} \\
\frac{2 m L^{2}}{J} \dot{\theta}
\end{array}\right)=\mathbf{q}(\theta, t)
$$


where $\mathbf{q}(\theta, t)$ is the control action

$$
\mathbf{q}(\theta, t)=\mathbf{P}(\theta) \mathbf{U}(t)
$$

with

$$
\mathbf{P}(\theta)=\left(\begin{array}{ccc}
-\sin \theta & -\sin \left(\frac{\pi}{3}-\theta\right) & \sin \left(\frac{\pi}{3}+\theta\right) \\
\cos \theta & -\cos \left(\frac{\pi}{3}-\theta\right) & -\cos \left(\frac{\pi}{3}+\theta\right) \\
1 & 1 & 1
\end{array}\right)
$$

\section{Restricting Admissible Controls}

Moving the robot from a point to another requires specifying the three voltages $U_{i}(t)$. Clearly, real-time optimal control of the differential equations (20) is not feasible with modest computational resources. There have been several attempts to overcome the complexity emerging from nonlinearity and coupling. d'Andréa-Novel et al. (1992) showed that dynamic feedback linearization can lead to the simplification of the control problem of 3 -wheeled robots. Time optimal trajectories were constructed by Balkcom and Mason (2000) for differential drive robots.

The goal of this section is to find a simplified, computationally tractable optimal control problem whose solution yields feasible, albeit sub-optimal, trajectories. This will be achieved by restricting the set of admissible controls.

The set of feasible voltages $\mathcal{U}$ is a cube given by

$$
\mathcal{U}(t)=\left\{\mathbf{U}(t)|\quad| U_{i}(t) \mid \leq 1\right\}
$$

The set of admissible controls $\mathbf{P}(\theta) \mathcal{U}(t)$ depends on the vehicle orientation $\theta$. Since $\theta$ is responsible for the coupling of equations (20), replacing this set with a set of $\theta$-independent controls would greatly simplify the problem. The maximal such set is found by taking the intersection of all possible sets of allowable controls

$$
\mathcal{Q}(t)=\bigcap_{\theta \in[0,2 \pi)} \mathbf{P}(\theta) \mathcal{U}(t)
$$

Obviously, any $\mathbf{q}(t) \in \mathcal{Q}$ is a suitable replacement for the $\theta$-dependent control action. In the following we give the explicit representation of $\mathcal{Q}$.

For a given $\theta$, the linear transformation $\mathbf{P}(\theta)$ maps the cube $\mathcal{U}(t)$ into the tilted cuboid (the set of allowable controls) $\mathbf{P}(\theta) \mathcal{U}(t)$. The matrix $\mathbf{P}(\theta)$ can be decomposed as a product of a rotation and a $\theta$-independent linear transformation

$$
\mathbf{P}(\theta)=\mathbf{R}_{z}(\theta) \mathbf{P}(0)
$$

where

$$
\mathbf{R}_{z}(\theta)=\left(\begin{array}{ccc}
\cos \theta & -\sin \theta & 0 \\
\sin \theta & \cos \theta & 0 \\
0 & 0 & 1
\end{array}\right), \quad \mathbf{P}(0)=\frac{1}{2}\left(\begin{array}{ccc}
0 & -\sqrt{3} & \sqrt{3} \\
2 & -1 & -1 \\
2 & 2 & 2
\end{array}\right)
$$

The linear transformation $\mathbf{P}(0)$ maps cube $\mathcal{U}(t)$ into the tilted cuboid $\mathbf{P}(0) \mathcal{U}(t)$ with a diagonal $\left|q_{\theta}\right| \leq 3$ along the $q_{\theta}$ axis (Figure 4 ). Note that since the mapping $\mathbf{P}(0)$ is linear, the surface of the cube gets mapped onto that of the cuboid. The transformation $\mathbf{R}_{z}(\theta)$ then rotates this cuboid about the $q_{\theta}$ axis (or equivalently: about its diagonal). The problem is to find the solid of revolution that is the intersection of all possible rotations $\mathbf{R}_{z}(\theta) \mathbf{P}(0) \mathcal{U}(t)$ of the cuboid. This solid of revolution is characterized by (see Appendix A for details)

$$
q_{x}^{2}(t)+q_{y}^{2}(t) \leq r^{2}\left(q_{\theta}(t)\right)
$$




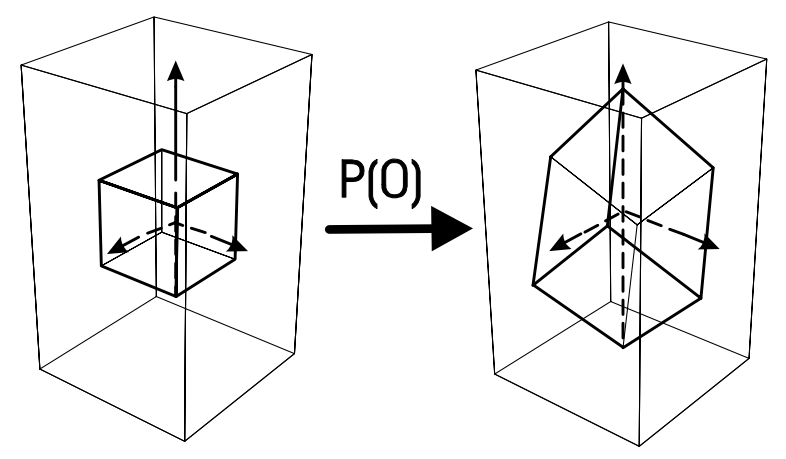

Figure 4: The mapping $P(0)$

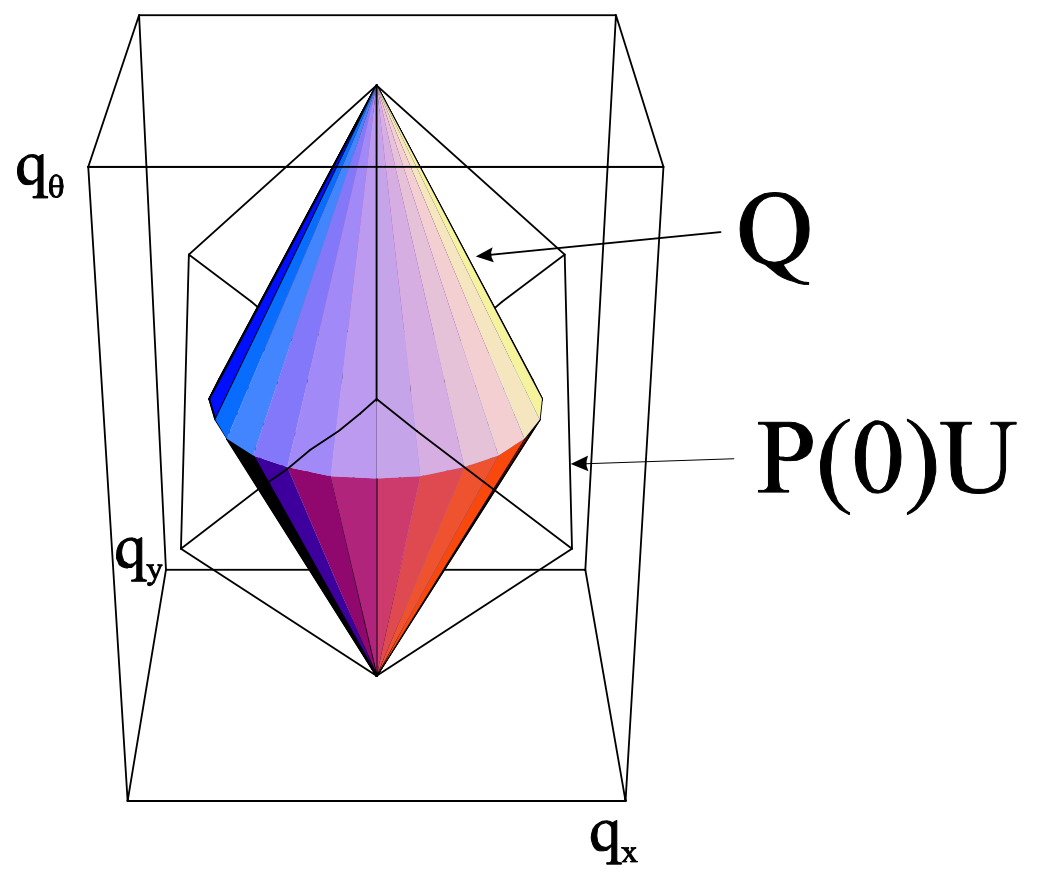

Figure 5: Restricted set for admissible controls

where the radius is

$$
r\left(q_{\theta}(t)\right)=\frac{3-\left|q_{\theta}(t)\right|}{2}
$$

The solid of revolution is illustrated in Figure 5, while its cross-section is shown in Figure 6 .

With this result the equations of motion decouple

$$
\begin{gathered}
\ddot{x}+\dot{x}=q_{x}(t) \\
\ddot{y}+\dot{y}=q_{y}(t) \\
\ddot{\theta}+\frac{2 m L^{2}}{J} \dot{\theta}=q_{\theta}(t)
\end{gathered}
$$

While these equations are linear, the control efforts are coupled, i.e. the constraints

$$
\begin{gathered}
q_{x}^{2}(t)+q_{y}^{2}(t) \leq\left(\frac{3-\left|q_{\theta}(t)\right|}{2}\right)^{2} \\
\left|q_{\theta}(t)\right| \leq 3
\end{gathered}
$$



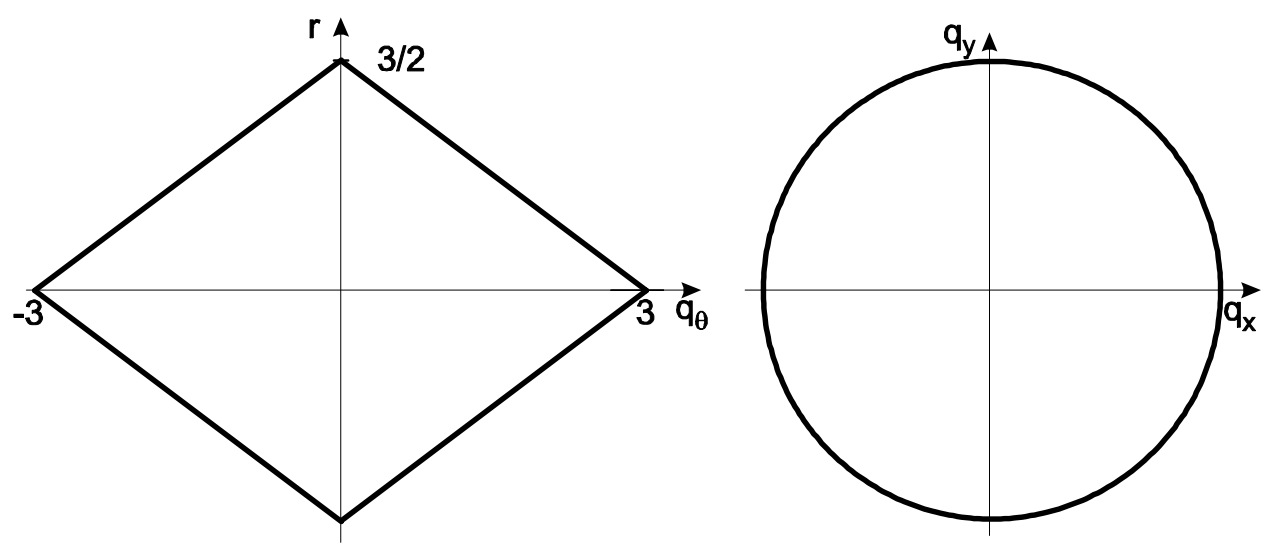

Figure 6: Cross-section of the admissible set

have to be satisfied.

In the remainder of the paper we focus our attention on controlling the translational DOFs. In particular, we will assume that the translational DOFs are controlled independently from the rotational DOF. The reasons for this are two-fold: 1) it substantially simplifies the exposition and development of the results, and the results can readily be extended to encompass all three DOFs simultaneously; 2) we envision that the main use of the results in this paper will be in applications where rotation control is independent of translation control.

To decouple the $\theta$-equation from those of the translational ones, we set

$$
\left|q_{\theta}(t)\right| \leq 1
$$

Then the constraint for $x$ and $y$ becomes

$$
q_{x}^{2}(t)+q_{y}^{2}(t) \leq 1
$$

Clearly other bounds on $q_{\theta}$ could be chosen.

\section{Trajectory Generation}

In this section we are concerned with finding a solution to the following system of linear equations

$$
\begin{aligned}
& \ddot{x}+\dot{x}=q_{x}(t) \\
& \ddot{y}+\dot{y}=q_{y}(t)
\end{aligned}
$$

with the boundary conditions

$$
\begin{aligned}
& x(0)=0, \quad x\left(t_{f}\right)=x_{f}, \quad \dot{x}(0)=v_{x 0}, \quad \dot{x}\left(t_{f}\right)=0 \\
& y(0)=0, \quad y\left(t_{f}\right)=y_{f}, \quad \dot{y}(0)=v_{y 0}, \quad \dot{y}\left(t_{f}\right)=0
\end{aligned}
$$

and the constraint on the control inputs

$$
q_{x}^{2}(t)+q_{y}^{2}(t) \leq 1
$$


The final time $t_{f}$ is the free variable that needs to be minimized. Note that the initial positions are assumed to be 0 , since this can always be achieved with a translation. The final velocities are specified to be zero. ${ }^{1}$

This system can also be written as

$$
\dot{\mathbf{z}}(t)=A \mathbf{z}(t)+B \mathbf{q}(t)
$$

where

$$
A=\left(\begin{array}{cccc}
0 & 1 & 0 & 0 \\
0 & -1 & 0 & 0 \\
0 & 0 & 0 & 1 \\
0 & 0 & 0 & -1
\end{array}\right), B=\left(\begin{array}{cccc}
0 & 0 & 0 & 0 \\
1 & 0 & 0 & 0 \\
0 & 0 & 0 & 0 \\
0 & 1 & 0 & 0
\end{array}\right), \mathbf{z}(t)=\left(\begin{array}{c}
x(t) \\
v_{x}(t) \\
y(t) \\
v_{y}(t)
\end{array}\right), \quad \mathbf{q}(t)=\left(\begin{array}{c}
q_{x}(t) \\
q_{y}(t) \\
0 \\
0
\end{array}\right)
$$

with the boundary conditions

$$
\mathbf{z}(0)=\left(\begin{array}{c}
0 \\
v_{x 0} \\
0 \\
v_{y 0}
\end{array}\right), \mathbf{z}\left(t_{f}\right)=\left(\begin{array}{c}
x_{f} \\
0 \\
y_{f} \\
0
\end{array}\right)
$$

subject to

$$
q_{x}^{2}(t)+q_{y}^{2}(t) \leq 1
$$

\subsection{Minimum Time Trajectory}

It is shown in Athans and Falb (1966) that the optimal control strategy is achieved when

$$
q_{x}^{2}(t)+q_{y}^{2}(t)=1 \quad t \in\left[0, t_{f}\right]
$$

Further, the time-optimal control is given by (\|.\| denotes the Euclidean norm)

$$
\mathbf{q}(t)=-\frac{B^{T} \mathbf{p}(t)}{\left\|B^{T} \mathbf{p}(t)\right\|}
$$

where $\mathbf{p}(t)$ is the solution to the adjoint problem

$$
\dot{\mathbf{p}}(t)=-A^{T} \mathbf{p}(t)
$$

The two components of the time-optimal control are thus

$$
\begin{aligned}
& q_{x}(t)=\frac{\lambda_{1}+e^{t-t_{f}}\left(\lambda_{2}-\lambda_{1}\right)}{\sqrt{\left(\lambda_{1}+e^{t-t_{f}}\left(\lambda_{2}-\lambda_{1}\right)\right)^{2}+\left(\lambda_{3}+e^{t-t_{f}}\left(\lambda_{4}-\lambda_{3}\right)\right)^{2}}} \\
& q_{y}(t)=\frac{\lambda_{3}+e^{t-t_{f}}\left(\lambda_{4}-\lambda_{3}\right)}{\sqrt{\left(\lambda_{1}+e^{t-t_{f}}\left(\lambda_{2}-\lambda_{1}\right)\right)^{2}+\left(\lambda_{3}+e^{t-t_{f}}\left(\lambda_{4}-\lambda_{3}\right)\right)^{2}}}
\end{aligned}
$$

where the parameters $\lambda_{i}, t_{f}$ can be determined from the boundary conditions $(38,39)$. The solution to system $(42,43)$ is

$$
\mathbf{z}(t)=e^{A t} \mathbf{z}(0)+\int_{0}^{t} e^{A(t-\tau)} B \mathbf{q}(\tau) d \tau
$$

\footnotetext{
${ }^{1}$ Note that specifying a non-zero final velocity together with a final position often leads to solutions that do not continuously depend on the boundary conditions, which is not a desirable property in most applications.
} 
with this, the boundary conditions are written as (note that the initial conditions are automatically satisfied)

$$
\begin{gathered}
x_{f}=v_{x 0}\left(1-e^{-t_{f}}\right)+\int_{0}^{t_{f}}\left(1-e^{\tau-t_{f}}\right) q_{x}(\tau) d \tau \\
0=e^{-t_{f}} v_{x 0}+\int_{0}^{t_{f}} e^{\tau-t_{f}} q_{x}(\tau) d \tau \\
y_{f}=v_{y 0}\left(1-e^{-t_{f}}\right)+\int_{0}^{t_{f}}\left(1-e^{\tau-t_{f}}\right) q_{y}(\tau) d \tau \\
0=e^{-t_{f}} v_{y 0}+\int_{0}^{t_{f}} e^{\tau-t_{f}} q_{y}(\tau) d \tau
\end{gathered}
$$

An additional equation can be obtained from the fact that the Hamiltonian of the system (see Athans and Falb, 1962) is zero on $\left[0, t_{f}\right]$.

$$
H=1+(A \mathbf{z}(t)+B \mathbf{q}(t), \mathbf{p}(t))=1-\lambda_{2} q_{x}\left(t_{f}\right)-\lambda_{4} q_{y}\left(t_{f}\right)=0
$$

where $(.,$.$) denotes scalar product. This is equivalent to$

$$
\lambda_{2}^{2}+\lambda_{4}^{2}=1
$$

The integrals in (51-54) can be obtained in closed form. Unfortunately, the resulting nonlinear equations (together with (56)) have to be solved numerically for the five unknowns. The authors could not find a computationally efficient method of solving this problem, both in the literature and using standard optimization packages. We will have more to say on this matter at the end of Section ??.

In order to gain numerical tractability, the problem is further relaxed by restricting the space of possible solutions to

$$
\left|q_{x}(t)\right|=\text { constant }, \quad\left|q_{y}(t)\right|=\text { constant }
$$

that is

$$
q_{z}(t)=\left\{\begin{array}{cc}
q_{z} & 0<t \leq t_{1} \\
-q_{z} & t_{1}<t \leq t_{f}
\end{array}\right.
$$

where $z$ represents either $x$ or $y$, and $q_{z} \in[-1,1]$ is a constant. It will be shown that these assumptions result in extremely computationally "cheap" solutions, and that the difference between the resulting $t_{f}$ and the optimal one is small.

The rest of the section is organized as follows: in Section 4.2 we construct optimal solutions separately for the translational degrees-of-freedom $x$ and $y$ without the coupling constraint (45). These separate solutions will in general yield different final times and so they must be synchronized. It will be shown in Section 4.3 that this can always be achieved and that the solution will also satisfy the required constraint (45). 


\subsection{Bang-bang Trajectory}

We first focus on solving the optimal control problem $(36,38)$ with the constraint

$$
\left|q_{z}(t)\right|=\text { constant }
$$

where $z$ represents either $x$ or $y$. This constant can always be taken as 1 by rescaling the equations. The minimum time problem consists of finding a solution with as small a $t_{f}$ as possible. It can be shown (for example Pierre, 1986), that this boundary value problem always has a solution, and that the control which minimizes the final time $t_{f}$ consists of two piecewise constant segments of magnitude 1. This type of control strategy is commonly referred to as "bang-bang" control. Koh and Cho (1999) formulated a path tracking problem for a twowheeled robot based on bang-bang control. In our case, the following must be solved for $q, t_{1}$ and $t_{2}$ :

$$
\begin{gathered}
\ddot{z}+\dot{z}=q_{z}, \quad 0<t \leq t_{1} \\
\ddot{z}+\dot{z}=-q_{z}, \quad t_{1}<t \leq t_{1}+t_{2}=t_{f}, \\
z(0)=0, \quad z\left(t_{f}\right)=z_{f}, \quad \dot{z}(0)=v_{0}, \quad \dot{z}\left(t_{f}\right)=0
\end{gathered}
$$

Subject to the boundary conditions $(62)$, equations $(60,61)$ can be solved to yield

$$
\begin{gathered}
z(t)=\left\{\begin{array}{cc}
e^{-t}\left(q_{z}-v_{0}\right)+q_{z}(t-1)+v_{0} & 0 \leq t<t_{1} \\
q_{z}\left(t_{f}-t-e^{t_{f}-t}+1\right)+z_{f} & t_{1} \leq t \leq t_{f}
\end{array}\right. \\
v(t)=\left\{\begin{array}{cc}
\left(v_{0}-q_{z}\right) e^{-t}+q_{z} & 0 \leq t<t_{1} \\
\left(e^{t_{f}-t}-1\right) q_{z} & t_{1} \leq t \leq t_{f}
\end{array}\right.
\end{gathered}
$$

Position and velocity should be continuous at $t=t_{1}$, that is

$$
\begin{gathered}
\left(q_{z}-v_{0}\right) e^{-t_{1}}+q_{z}\left(t_{1}-1\right)+v_{0}=-e^{t_{2}} q_{z}+q_{z}\left(1+t_{2}\right)+z_{f} \\
\left(v_{0}-q_{z}\right) e^{-t_{1}}+q=q_{z}\left(e^{t_{2}}-1\right)
\end{gathered}
$$

These conditions are equivalent to

$$
\begin{gathered}
t_{1}=t_{2}-c / q_{z} \\
q_{z}\left(e^{t_{2}}\right)^{2}-2 q_{z} e^{t_{2}}+\left(v_{0}-q_{z}\right) e^{c / q_{z}}=0
\end{gathered}
$$

where $c=v_{0}-z_{f}$. The second equation can be solved as

$$
e_{1,2}^{t_{2}}=1 \pm \operatorname{sgn}\left(q_{z}\right) \sqrt{D}
$$

where

$$
D=1+e^{c / q_{z}}\left(\frac{v_{0}}{q_{z}}-1\right)
$$

We also require $t_{1} \geq 0, t_{2} \geq 0$ which will then provide

$$
t_{2}=\ln (1+\sqrt{D})
$$

The following inequalities should hold

$$
\begin{gathered}
D \geq 0 \\
\sqrt{D} \geq e^{\frac{c}{q_{z}}}-1
\end{gathered}
$$


If $c / q_{z} \leq 0$ then (72) has to be true, that is

$$
e^{-\frac{c}{q_{z}}}-1 \geq-\frac{v_{0}}{q_{z}}
$$

If $c / q_{z} \geq 0$ then

$$
D \geq\left(e^{\frac{c}{q z}}-1\right)^{2}
$$

should be satisfied, which is equivalent to

$$
e^{\frac{c}{q_{z}}}-1 \leq \frac{v_{0}}{q_{z}}
$$

Multiplying (74) and (76) by $c / q_{z}$ (and taking its sign into account) yields

$$
\frac{c}{q_{z}}\left(e^{\left|\frac{c}{q z}\right|}-1\right) \leq\left|\frac{c}{q_{z}}\right| \frac{v_{0}}{q_{z}}
$$

which can be further simplified to

$$
\operatorname{sgn} \frac{c}{q_{z}}\left(e^{\left|\frac{c}{q_{z}}\right|}-1\right) \leq \frac{v_{0}}{q_{z}}
$$

The sign of the first segment $( \pm 1)$ is thus given by

$$
q_{z}=\operatorname{sgn}\left(v_{0}-\operatorname{sgn}(c)\left(e^{|c|}-1\right)\right)
$$

Once $q_{z}, t_{1}$ and $t_{2}$ are determined, $z(t)$ is given by (63). The execution time for this trajectory (using $(67,71))$ is

$$
t_{f \min }:=t_{1}+t_{2}=2 \ln (1+\sqrt{D})-c / q_{z}
$$

\subsection{Trajectory Synchronization}

Generally, execution times for the minimum time problems for the different degrees of freedom will be different. To find a solution to the boundary value problem $(36,37,38,39)$, these solutions must be synchronized, that is $t_{f x}=t_{f y}$ should hold. If we could vary the execution time for the different degrees of freedom, this synchronization might be possible. The question naturally arises: does a bang-bang trajectory exist for a fixed time $t_{f}>t_{f \min }$ ? Since the boundary conditions are given, we only have control over the control effort. If the control effort $\bar{q}=\left|q_{z}\right|$ is decreased one intuitively expects the execution time $t_{f}$ to increase. The following proposition is proved in Appendix B:

Proposition 1 For all $t_{f} \geq t_{\text {fmin }}$ there exists a $\bar{q} \in(0,1]$ such that

$$
q_{z}=\bar{q} \operatorname{sgn}\left(\frac{v_{0}}{\bar{q}}-\operatorname{sgn}(c)\left(e^{\left|\frac{c}{\bar{q}}\right|}-1\right)\right)
$$

satisfies (60, 61). Furthermore, the execution time is a continuous, strictly monotonously decreasing function of $\bar{q}$ with $\lim _{\bar{q} \rightarrow 0} t_{f} \rightarrow \infty$ and $t_{f}(\bar{q}=1)=t_{\text {fmin }}$.

This result means that reaching the desired final position in the prescribed amount of time (provided that this time is greater then the minimum time to reach this position with zero final velocity) is always possible with a reduced effort bang-bang control strategy. Note that the execution time depends on the boundary conditions, as well as on the control effort, i.e.

$$
t_{f z}\left(\left|q_{z}\right|\right)=t_{f}\left(z_{f}, v_{z 0}, q_{z}\right)
$$


With this notation, we want to find the control efforts $q_{x}$ and $q_{y}$ for which

$$
t_{f x}\left(\left|q_{x}\right|\right)=t_{f y}\left(\left|q_{y}\right|\right)
$$

Using the constraint (45) this is written as

$$
t_{f x}\left(\left|q_{x}\right|\right)=t_{f y}\left(\left|\sqrt{1-q_{x}^{2}}\right|\right)
$$

Since $t_{f x}\left(\left|q_{x}\right|\right)$ is a strictly monotonously decreasing function on $\left|q_{x}\right| \in(0,1], t_{f y}\left(\left|\sqrt{1-q_{x}^{2}}\right|\right)$ is strictly monotonously increasing there (and thus $t_{f x}\left(\left|q_{x}\right|\right)-t_{f y}\left(\left|\sqrt{1-q_{x}^{2}}\right|\right)$ is strictly monotonously decreasing). Hence there exists a unique $\left|q_{x}\right|$ satisfying (84). Furthermore, these control efforts satisfy the constraint (45) and thus yield the solution of the boundaryvalue problem $(36,37,38,39,45)$. This solution will also depend continuously on the boundary conditions (see Appendix B) which ensures robustness to disturbances.

\section{$5 \quad$ Numerical Solutions and Simulations}

To demonstrate the computational efficiency and robustness of the algorithm, simulations were performed $^{2}$ (with $\alpha=1 \mathrm{~N} / \mathrm{V}$ and $\beta=1 \mathrm{~kg} / \mathrm{s}$ ). Position and velocity of the vehicle are assumed to be measured sixty times a second $(d t=0.017 \mathrm{~s})$. The inputs to the algorithm are initial and desired positions and velocities $\left(x_{0}, y_{0}, v_{x 0}, v_{y 0}, x_{f}, y_{f}\right)$ and the outputs are the velocities $\left(v_{x}, v_{y}\right)$ that should be reached in the next timestep. The inputs are first translated (so that $z_{0}^{\text {new }}=0, z_{f}^{\text {new }}=z_{f}-z_{0}$ ) then nondimensionalized (by (19)). The control efforts are found by performing bisections on the $q_{x}$ axis to find the zero of $t_{f x}\left(\left|q_{x}\right|\right)-t_{f y}\left(\left|\sqrt{1-q_{x}^{2}}\right|\right)$ (this strictly monotonic quantity goes to positive infinity for $q_{x} \rightarrow 0$ and negative infinity as $q_{x} \rightarrow 1$, so care must be taken with the bounds). The bisection algorithm is stopped when the function value is less than $d t$. With $q_{x}, q_{y}$ the positions and velocities after a timestep can be calculated as (see (63) and (64))

$$
\begin{gathered}
z=e^{-d t}\left(q_{z}-v_{z 0}\right)+q_{z}(d t-1)+v_{z 0} \\
v_{z}=\left(v_{z 0}-q_{z}\right) e^{-d t}+q_{z}
\end{gathered}
$$

These nondimensional quantities should then be scaled back to dimensional ones.

A representative simulation is now discussed, where the following initial and final conditions were used

$$
\begin{aligned}
x_{0} & =y_{0}=0 \mathrm{~m}, \quad x_{f}=y_{f}=1 \mathrm{~m} \\
v_{x 0} & =0.2 \frac{\mathrm{m}}{\mathrm{s}}, \quad v_{y 0}=-0.5 \frac{\mathrm{m}}{\mathrm{s}}
\end{aligned}
$$

The position and velocity of the vehicle is calculated by (85) and (86). To account for errors present in a real system (arising from slippage, measurement errors, etc.) noise was added to the actual position and velocity of the robot at the end of every simulation step. The disturbances were modeled as white noise, with amplitude of $1 \mathrm{~cm}$ and $3 \mathrm{~cm} / \mathrm{s}$ from a uniform distribution for positions and velocities, respectively. This was repeated until both coordinates were within 5 centimeters of the target position and the velocities were less than $5 \mathrm{~cm} / \mathrm{s}$ in absolute value. The results are shown in Figure 7. This closed-loop trajectory is close to the trajectory generated at $t=0$ (the open-loop trajectory), showing the robustness of the proposed method. Note that the most pronounced effect of the noise on the trajectory is in the vicinity of the destination. The position error coupled with the discretization effect gives rise to jerky motion. This can be avoided for example by switching to open-loop control near the target, or by simply stopping.

\footnotetext{
${ }^{2}$ The code used to generate these results are available at www.tam.cornell.edu/ $\sim$ nagy/omni.html
} 


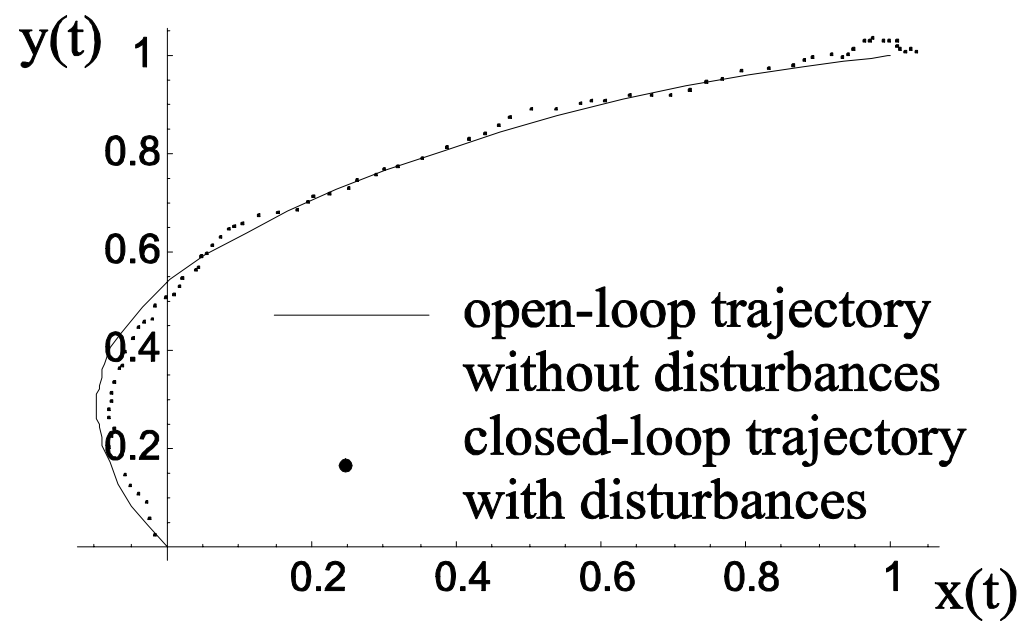

Figure 7: Simulation with noise

For this particular example, the difference between the approximate bang-bang solution and the optimal trajectory is very small. Figure 8 shows the piecewise constant bang-bang solution compared to the optimal one.

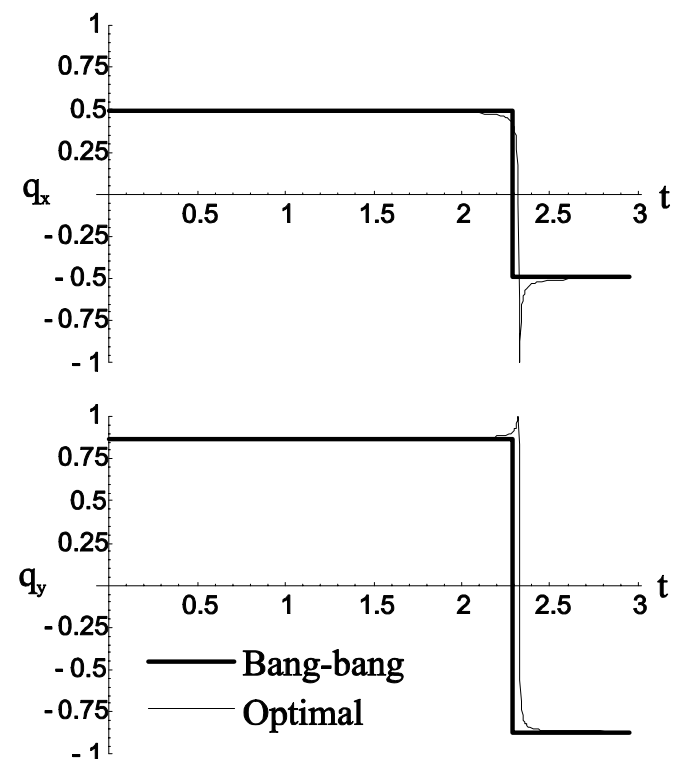

Figure 8: Comparison of optimal and approximate solution

To demonstrate how well the optimal solutions are approximated by our solutions, 1000 simulations were performed with randomly generated initial velocities $(\leq 1 \mathrm{~m} / \mathrm{s}$ in magnitude) and final positions ( $\leq 3 \mathrm{~m}$ in magnitude) from uniform distributions. The difference between the optimal solution and the reduced effort bang-bang solutions is shown in Table 1. 


$\begin{array}{cc}t_{\text {fopt }} / t_{f} \text { is less than } & \text { percentage of problems } \\ 99.9 \% & 16.4 \% \\ 99.5 \% & 2.7 \% \\ 99 \% & 1.3 \% \\ 97.4 \% & 0 \%\end{array}$

Table 1. Comparison of optimal and near-optimal solutions.

For example, the optimal solution was 1 percent or more better than than the bang-bang solution in only 1.3 percent of the random examples; in none of the 1000 random examples did the optimal solution yield an improvement of more than 2.6 percent. The computational cost of the proposed algorithm is extremely low, approximately 300 FLOPS (floating point operations) for one timestep. The coefficients of the optimal solution (that is equations (51$54,56)$ ) were numerically solved for in Matlab with a computational cost of approximately 50,000 FLOPS.

\section{Conclusions}

The main benefit of the omnidirectional drive mechanism is a simplification of the resulting control problem, which greatly reduces the computation required for generating nearly optimal trajectories. The proposed algorithms provide an efficient, yet high performance, method of path planning. The algorithms are in general conservative; this conservatism is small, however, and is justified by the extremely reduced computational costs. The extremely low computational cost means that these nearly optimal trajectories can be used extensively as low overhead primitives by higher level decision making strategies, allowing a large number of possible scenarios to be explored in real time. For example, these trajectory primitives can readily be used as a basis for obstacle avoidance in randomized path-planning algorithms (see for example Frazzoli et al., 2002). 


\section{Appendix A}

Figure 9 shows the cuboid. The radius of the largest contained solid of revolution at a fixed $q_{\theta}$ is simply the radius of the biggest circle that can be inscribed into the polygonal intersection of the cuboid and the plane $q_{\theta}(t)=$ constant. (Figure 9 also shows this circle at $q_{\theta}(t)=1$ ). Because of the symmetry of the cuboid it is enough to study the plane containing the line

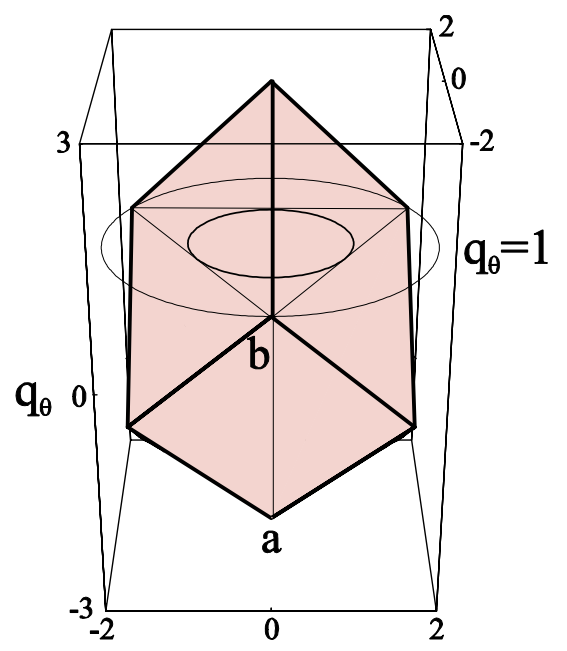

Figure 9: Geometry of the cuboid

segment $a b$. It is easy to see that the radius sought is just the distance of this line from the $q_{\theta}$ axis at a given height. The segment $a b$ connects point $(0,0,-3)$ and $(0,-2,1)$ so its equation is

$$
q_{y}(t)=\frac{3+q_{\theta}(t)}{2}, \quad q_{x}(t)=0 \quad-3 \leq q_{\theta}(t) \leq 1
$$

The solid of revolution is thus characterized by

$$
q_{x}^{2}(t)+q_{y}^{2}(t) \leq\left(\frac{3-\left|q_{\theta}(t)\right|}{2}\right)^{2}=r^{2}\left(q_{\theta}(t)\right)
$$




\section{Appendix B}

To prove Proposition 1 we first define

$$
w=\frac{1}{2 q}
$$

Then $t_{2}$ can be expressed as

$$
t_{2}=\frac{t_{f}}{2}+c w
$$

With $(67,92)$, equation (66) can be recast as

$$
e^{-c w}=\cosh \left(\frac{t_{f}}{2}\right)-v_{0} e^{-\frac{t_{f}}{2}} w
$$

Figure 10 shows the left and right hand side of equation (94) as a function of $w$ for the case of $c>0$.

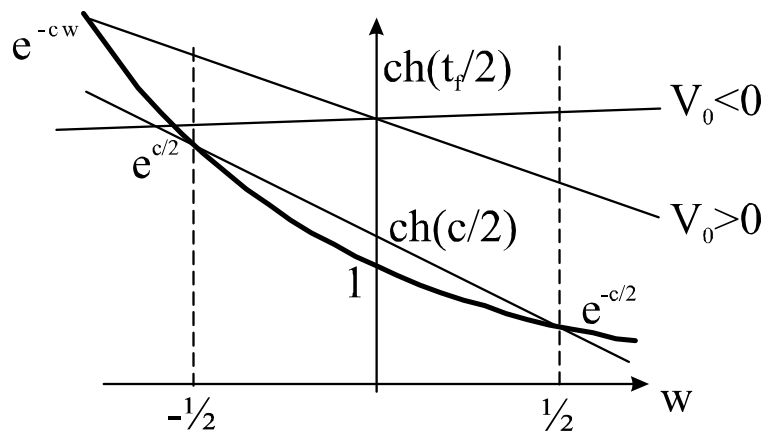

Figure 10: Solution to the fixed-time problem

Proposition 2 For all $t_{f}>t_{f m i n}$ there exists a $w$ that satisfies

$$
e^{-c w}=\cosh \left(\frac{t_{f}}{2}\right)-v_{0} e^{-\frac{t_{f}}{2}} w
$$

and $|w|>\frac{1}{2}$. Further, the execution time $t_{f}$ is a strictly monotonous function of $q$ with $\lim t_{f} \rightarrow \infty$ as $q \rightarrow 0$.

Proof: First we show that

$$
t_{f} \geq|c|
$$

or equivalently

$$
\cosh \left(\frac{t_{f}}{2}\right) \geq \cosh \left(\frac{c}{2}\right)
$$

To see this, consider the inequalities

$$
\begin{gathered}
t_{f}=t_{1}+t_{2} \geq t_{2} \\
t_{1}=t_{2}-\frac{c}{q} \geq 0 \Rightarrow t_{2} \geq \frac{c}{q}
\end{gathered}
$$

if $\operatorname{sgn}\left(\frac{c}{q}\right)=1$

$$
\frac{c}{q}=|c| \Rightarrow t_{2} \geq|c| \Rightarrow t_{f} \geq|c|
$$


if $\operatorname{sgn}\left(\frac{c}{q}\right)=-1$

$$
t_{f}=\ln (1+\sqrt{D})^{2}-\frac{c}{q}=\ln (1+\sqrt{D})^{2}+|c| \geq|c|
$$

since $D \geq 0$.

Using this we are able to show the existence of roots with $|w|>\frac{1}{2}$.

If $v_{0}<0$ then the slope of the line

$$
\cosh \left(\frac{t_{f}}{2}\right)-v_{0} e^{-\frac{t_{f}}{2}} w \quad t_{f}>t_{f \min }
$$

will decrease, so the $w$ coordinate of its intersection with the exponential function will always be less than $-\frac{1}{2}$.

If $v_{0}>0$ then the line (101) will have one intersections with the exponential function $e^{-c w}$, for which

$$
w>w^{*}=\frac{1}{2} \text { or } w<w^{*}=-\frac{1}{2}
$$

If $e^{\frac{c}{2}} \leq \cosh \left(\frac{t_{f}}{2}\right)$ then the line $(101)$ and $e^{-c w}$ will have one intersection (since the positive slope of the line is decreasing with increasing $t_{f}$ ) with

$$
w<-\frac{1}{2}
$$

These arguments also shows that increasing $|w|$ corresponds to increasing $t_{f}$ (that is $|w|$ $(|q|)$ is a monotously increasing (decreasing) function of $t_{f}$ ). The converse is also true: $t_{f}$ is a strictly monotonously increasing function of $q$ with $\lim t_{f} \rightarrow \infty$ as $q \rightarrow 0$. The $c<0$ case can similarly be proved. This concludes the proof.

\section{References}

[1] Asada, M. and Kitano, H., editors, RoboCup-98: Robot Soccer World Cup II, In Lecture Notes in Computer Science, Springer, 1999.

[2] Athans, M. and Falb, P. L., Optimal Control: An introduction to the theory and its applications., McGraw-Hill, New York, 1966.

[3] d'Andréa-Novel, B., Bastin, G. and Campion, G., Dynamic feedback linearization of nonholonomic wheeled mobile robots, in Proceedings of the IEEE International Conference on Robotics and Automation, 3, pp. 2527-2532, 1992.

[4] D'Andrea, R., Kalmár-Nagy, T., Ganguly, P., Babish, M.: The Cornell Robocup Team, in Kraetzschmar, G., Stone P., Balch T. editors: Robot Soccer WorldCup IV, Lecture Notes in Artificial Intelligence. Springer, Berlin, Heidelberg, New York, 2001.

[5] Balkcom, D. and Mason, M. Time Optimal Trajectories for Bounded Velocity Differential Drive Robots, in Proceedings of the IEEE International Conference on Robotics and Automation (ICRA '00), 3, pp. 2499 - 2504, 2000.

[6] Faiz, N. and Agrawal, S. K., Trajectory planning of robots with dynamics and inequalities in Proceedings of the 2000 IEEE International Conference on Robotics and Automation, 4, pp. 3976-3982, 2000.

[7] Frazzoli E., Dahleh, M.A. and Feron E., Real-Time Motion Planning for Agile Autonomous Vehicles, AIAA Journal of Guidance, Control, and Dynamics, 25(1), pp. 116129, 2002. 
[8] Jung, M., Shim, H., Kim, H. and Kim, J., The Miniature Omni-directional Mobile Robot OmniKity-I (OK-I), in Proceedings of the International Conference on Robotics and Automation, 4, pp. 2686-2691, 1999.

[9] Kitano, H., editor, RoboCup-97: Robot Soccer World Cup I, In Lecture Notes in Computer Science, Springer, 1998.

[10] Koh, K. C. and Cho., H. S., A Smooth Path Tracking Algorithm for Wheeled Mobile Robots with Dynamic Constraints, Journal of Intelligent and Robotic Systems, 24, pp. 367-385, 1999.

[11] Moore, K. L. and Flann, N. S., Hierarchial Task Decomposition Approach to Path Planning and Control for an Omni-Directional Autonomous Mobile Robot, in Proceedings of the International Symposium on Intelligent Control/Intelligent Systems and Semiotics, pp. 302-307, 1999.

[12] Muñoz, V., Ollero, A., Prado, M., Simón, A., Mobile robot trajectory planning with dynamics and kinematics constraints, in Proceedings of the IEEE International Conference on Robotics and Automation, pp. 342-351, 1994.

[13] Pierre, D. A., Optimization Theory with Applications. Dover, second edition, 1986.

[14] Pin, F. G. and Killough, S. M., A New Family of Omnidirectional and Holonomic Wheeled Platforms for Mobile Robots, In IEEE Transactions on Robotics and Automation, 10(4), pp. 480-489, 1994.

[15] Pontryagin, L. S., Boltyanskii, V. G., Gamkrelidze, R. V., Mischenko, E. F., The Mathematical Theory Of Optimal Processes. InterScience, 1962.

[16] Renaud, M. and Fourquet, J. Y., Minimum time motion of a mobile robot with two independent, acceleration-driven wheels, In Proceedings of the 1997 IEEE, International Conference on Robotics and Automation, pp. 2608-2613, 1997.

[17] Tanaka, Y., Tsuji, T., Kaneko, M., Morasso, P.G., Trajectory generation using time scaled artificial potential field, in Proceedings of the 1998 IEEE/RSJ International Conference on Intelligent Robots and Systems, 1, pp. 223-228, 1998.

[18] Veloso, M., Pagello, E. and Kitano, H., editors, RoboCup-99: Robot Soccer World Cup III, In Lecture Notes in Computer Science. Springer, 2000.

[19] Watanabe, K., Shiraishi, Y., Tzafestas, S. G., Tang, J. and Fukuda, T., Feedback Control of an Omnidirectional Autonomous Platform for Mobile Service Robots, Journal of Intelligent and Robotic Systems, 22(3), pp. 315-330, 1998. 